\title{
Aging studies for the ATLAS Transition Radiation Tracker (TRT)
}

T. Akesson ${ }^{\text {a }}$, E. Barberio $^{\text {b }}$, V. Bondarenko ${ }^{c}$, M. Capeans $^{b}$,

A. Catinaccio ${ }^{\text {b }}$, P. Cwetanski ${ }^{b}$, H. Danielsson ${ }^{\text {b }}$, F. Dittus ${ }^{b}$, B. Dolgoshein ${ }^{\mathrm{c}}$, N. Dressnandt ${ }^{\mathrm{d}}$, W. L. Ebenstein ${ }^{\mathrm{e}}$, P. Eerola $^{\mathrm{a}}$, P. Farthouat ${ }^{b}$, O. Fedin ${ }^{\text {f }}$, D. Froidevaux ${ }^{\text {b }}$, I. Gavrilenko ${ }^{\mathrm{g}}$, Y. Grichkevitch ${ }^{\text {h }}$, P. Gagnon ${ }^{i}$, Z. Hajduk ${ }^{j}$, P. T. Keener ${ }^{d}$, G. Kekelidze ${ }^{\mathrm{k}}$, S. Konovalov ${ }^{\mathrm{g}}$, T. Kowalski ${ }^{\ell}$, V. A. Kramarenko ${ }^{\text {h }}$, A. Laritchev ${ }^{h}$, P. Lichard ${ }^{\text {b }}$, B. Lundberg ${ }^{\text {a }}$, F. Luehring ${ }^{\mathrm{i}}$, I. Markina ${ }^{\mathrm{c}}$, A. Manara ${ }^{\mathrm{i}}, \mathrm{K}$. Mcfarlane ${ }^{\mathrm{m}}$, V. Mitsou ${ }^{\text {b }}$, S. Muraviev ${ }^{g}$, F. M. Newcomer ${ }^{d}$, H. Ogren ${ }^{i}$, S. H. Oh ${ }^{\mathrm{e}}$, J.Olszowska ${ }^{\mathrm{j}}$, V. Peshekhonov ${ }^{\mathrm{k}}$, C. Rembser ${ }^{\mathrm{b}}$, A. Romaniouk ${ }^{c, b, *}$, O. Rhone ${ }^{b}$, D. R. Rust ${ }^{i}$, V. Schegelsky ${ }^{f}$, A. Shmeleva ${ }^{g}$, S. Smirnov ${ }^{\text {c }}$, L. N. Smirnova ${ }^{\text {h }}$, V. Sosnovtsev ${ }^{\text {c }}$, S. Sutchkov ${ }^{\text {c }}$, F. Tartarelli ${ }^{\text {b }}$, V. Tikhomirov ${ }^{g}$, R. Van Berg ${ }^{d}$, L. Vassilieva ${ }^{\mathrm{g}}$, C. Wang ${ }^{\mathrm{e}}$, H. H. Williams ${ }^{\mathrm{d}}$

\section{Presented by Anatoli Romaniouk}

${ }^{a}$ Fysiska Institutionen, Lunds Universitet, Lund, Sweden

${ }^{\mathrm{b}}$ European Laboratory for Particle Physics (CERN), Geneva, Switzerland

${ }^{\mathrm{c}}$ Moscow Engineering and Physics Institute, Moscow, Russia

${ }^{\mathrm{d}}$ Department of Physics and Astronomy, University of Pennsylvania, Philadelphia, Pennsylvania, USA

e Physics Department, Duke University, Durham, North Carolina, USA

${ }^{\mathrm{f}}$ Petersburg Nuclear Physics Institute, Gatchina, St. Petersburg, Russia g P.N.Lebedev Institute of Physics, Moscow, Russia

${ }^{\mathrm{h}}$ Moscow State University, Institute of Nuclear Physics, Moscow, Russia

${ }^{\mathrm{i}}$ Indiana University, Bloomington, Indiana, USA

${ }^{\mathrm{j} H e n r y k}$ Niewodniczanski Institute of Nuclear Physics, Cracow, Poland

${ }^{\mathrm{k}}$ Joint Institute of Nuclear Research, Dubna, Russia

${ }^{\ell}$ Faculty of Physics and Nuclear Techniques of the Academy of Mining and Metallurgy, Cracow, Poland

${ }^{\mathrm{m}}$ Hampton University, Hampton, USA 


\begin{abstract}
A summary of the aging and material validation studies carried out for the ATLAS Transition Radiation Tracker (TRT) is presented. Particular emphasis is put on the different phenomena observed in straw tubes operating with the chosen $\mathrm{Xe} / \mathrm{CF}_{4} / \mathrm{CO}_{2}$ mixture. The most serious effects observed are silicon deposition on the anode wire and damage of the anode wire gold plating. Etching phenomena and active radical effects are also discussed. With a careful choice of all materials and components, and with good control of the water contamination in the active gas, the ATLAS TRT will operate reliably for 10 years at the LHC design luminosity. To demonstrate this fully, more work is still needed on the gas system purification elements, in particular to understand their interplay with the active species containing fluorine created in the avalanche process under irradiation.
\end{abstract}

Key words: TRT, Proportional chamber, Straw, Aging, Gold damage, Etching, $\mathrm{CF}_{4}$

\title{
1 Introduction
}

One of three major components of the ATLAS Inner Detector is the Transition Radiation Detector/Tracker (TRT) [1]. The TRT combines charged particle track reconstruction with electron identification capability. This is achieved by interleaving the radiator with xenon-filled thin-wall drift tubes of $4 \mathrm{~mm}$ diameter (straws). The total number of straws in the TRT is 372,032 with a length of about $40 \mathrm{~cm}$ (end-cap wheels with radial straws) and $150 \mathrm{~cm}$ (barrel modules with axial straws).

The TRT is situated close to the proton-proton interaction point (the detector extends between radii of $56 \mathrm{~cm}$ and $107 \mathrm{~cm}$ ) and the particle fluxes through the straws are therefore very high, as shown in Table 1 . After 10 years of LHC operation, the TRT straws will have accumulated up to 10 MRad of radiation dose and up to $2 \cdot 10^{14} \mathrm{n} / \mathrm{cm}^{2}$ in terms of neutron fluence. As shown in Table 1, the resulting current and accumulated charge are unprecedented and have resulted in a 12 -year $R \& D$ programme, which is not completed yet.

For such a huge and complex experiment as ATLAS, access to any part of the detector (particularly to the Inner Detector) will be very limited and all the components must be designed to be extremely reliable and the detectors

* Corresponding author. CERN, CH 1211,Geneve 23, Switzerland. Tel: +41-22-76711-76, fax: +41-22-767-83-50, e-mail: Anatoli.Romaniouk@cern.ch 
Table 1

Operating conditions of the ATLAS TRT at LHC

Charged particle flux up to

Neutron flux up to

Photon flux up to

Counting rate per wire up to

Ionisation current density up to

Ionisation current per wire up to

Power dissipated by ionisation current per straw up to

Charge collected over 10 years of LHC operation up to

Total charge per straw collected over 10 years of LHC operation up to

Total ionisation current in the detector volume

Total dissipating energy in the detector volume from ionising particles
$5 \cdot 10^{5} / \mathrm{cm}^{2} / \mathrm{s}$

$3 \cdot 10^{6} / \mathrm{cm}^{2} / \mathrm{s}$

$10^{7} / \mathrm{cm}^{2} / \mathrm{s}$

$20 \mathrm{MHz}$

$0.15 \mu \mathrm{A} / \mathrm{cm}$

$10 \mu \mathrm{A}$

$15 \mathrm{~mW}$

$10 \mathrm{C} / \mathrm{cm}$

$1500 \mathrm{C}$

$3 \mathrm{~A}$

$5 \mathrm{~kW}$

themselves must be very robust to ensure stable operation over more than 10 years. During the design and development studies of the TRT, particular attention was paid to the choices for the detecting elements and for the materials of the mechanical structure. A short description of the results of the validation process for these two items is presented in Section 2 and Section 3. Standard aging effects such as silicon deposits on the wire surface are discussed in Section 4. During these studies, a new phenomenon, damage of the anode wire gold coating, was observed and studied (Section 5). For $\mathrm{CF}_{4}$-containing mixtures, an important issue is the creation of active radicals in the avalanche plasma. These species have been shown to attack materials not only in the avalanche region but also downstream in the direction of the gas flow. This problem is discussed in Section 6, and the main conclusions of the studies achieved to-date are drawn in Section 7.

\section{Gas and straw cathode choice}

After detailed studies carried out within the TRT collaboration [2-5], an active gas mixture of $70 \% \mathrm{Xe}+20 \% \mathrm{CF}_{4}+10 \% \mathrm{CO}_{2}$ was chosen for the straw tube operation at the LHC. The straw tubes will be operated at a gas gain of $2.5 \cdot 10^{4}$ corresponding to a high voltage of $1530 \mathrm{~V}$. All the measurements reported in this paper, unless explicitly mentioned otherwise, were carried out with straws operating at this voltage. Obviously, for irradiation with high current densities, space-charge effects will reduce the actual gas gain in the irradiation 
area. The chosen gas mixture combines the advantages of efficient transition radiation absorption, short electron drift time, a wide operation plateau, and minimum aging effects in contrast to mixtures containing hydro-carbons. It should be stressed that the operational stability of the straw tubes is very much defined by this combination of $\mathrm{CF}_{4}$ and $\mathrm{CO}_{2}$ as additives to the $\mathrm{Xe}$ gas. Any binary mixture $\left(\mathrm{Xe} / \mathrm{CO}_{2}\right.$ or $\left.\mathrm{Xe} / \mathrm{CF}_{4}\right)$ would have a much narrower operational plateau.

The TRT straw tube is a cylindrical proportional chamber, with a gold-plated tungsten anode wire with a diameter of $30 \mu \mathrm{m}$. The straw wall (cathode) with a diameter of $4 \mathrm{~mm}$ is produced from a coated polyimide (Kapton) film. The electrical conductivity is assured by a thin aluminium layer of about $0.1 \mu \mathrm{m}$ thickness. This layer is protected against erosion during straw operation by a conductive composite layer of Carbon (30\%) and polyimide of approximately $6 \mu \mathrm{m}$ thickness $[2,3]$.

The electro-chemical reactions occurring in the straws operating under irradiation produce active species, which may attack the cathode surface and cause damage. In order to define the final specifications of the coating procedure for the TRT straw production, we carefully studied the cathode robustness up to very large irradiation doses. Micro-photographs of samples of coated film produced according to the final specifications are shown in Figs. 1 and 2, respectively, before and after irradiation $(18 \mathrm{C} / \mathrm{cm}$ total accumulated charge).

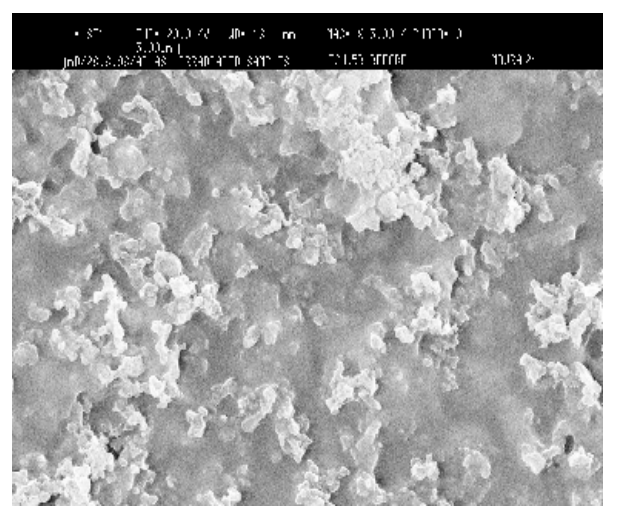

Fig. 1. Micro-photograph of the cathode surface before irradiation $(25 \times 20 \mu \mathrm{m})$.

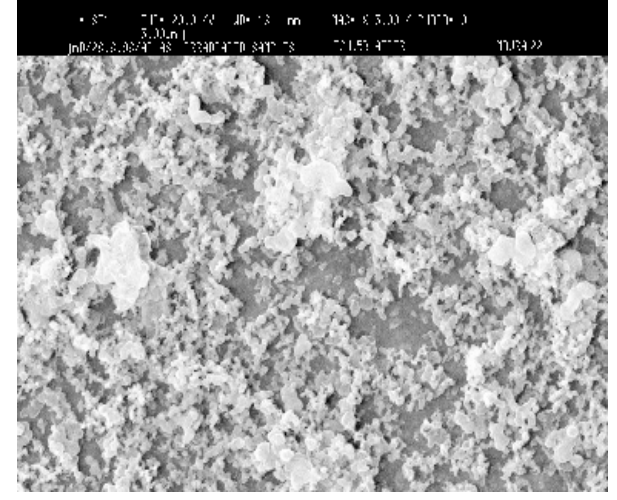

Fig. 2. Micro-photograph of the cathode surface after irradiation $(25 \times 20 \mu \mathrm{m})$.

Only slightly visible changes of the cathode were observed. A detailed massspectrometer analysis showed a small reduction of the relative oxygen concentration. This indicates that the amount of polyimide in the protection layer after irradiation has decreased, but most of the Carbon is still present to protect the aluminium layer, which shows no sign of damage. It is important also to note that no other effects such as deposits on the wire, sparks nor increased dark currents have been observed, when operating the straws after this amount of irradiation. 


\section{Detector material studies}

The aging processes depend not only on the active gas composition but also on the presence or not of even very minute traces of contaminations, either initially present in the gas mixture, or appearing at a later stage as a result of outgassing from detector materials or of gas-system components. Materials which are in direct contact with the active gas may have dramatic effects on the life-time of gaseous detectors. The materials incorporated into the TRT detector were chosen and validated in a series of steps:

a) In a first step a preliminary selection of materials was performed on the basis of their known chemical, mechanical, electrical, radiation hardness and outgassing properties. The detailed list of materials and their properties resulting from this step can be found in [6].

b) In a second step, the preselected materials were tested for outgassing before and after irradiation (integral dose of $50 \mathrm{MRad}$ ). The set-up contained a gas supply line, a box containing the materials that allowed to heat the samples up to $65^{\circ} \mathrm{C}$, and a gas chromatograph with a mass-spectrometer.

The preselected materials, which were tested in this way, are listed below:

- Glues: Araldite (AW 106, AW 134, AW 103), Redux 402, Rutapox L20, Stycast 1266, Trabond 2115, Traduct 2902 and Pronto;

- Materials: various CFRP materials, various plastic materials (Ultem, PEEK, Polycarbonate), and various printed circuit boards based on FR4 and G10, as produced and after some additional machining (including flex-rigid boards containing polyimide, solder mask, and different coverlays), etc.

A complete description of these tests is given in [7]. Only Araldite AW 106 was rejected after these tests because it displays a large amount of outgassing before and after irradiation. This step cannot, however, be considered as a final validation step because the outgassing measurement devices (gas chromatograph and mass-spectrometer) do not have enough sensitivity for the minute quantities of contaminants, which are nevertheless sometimes sufficient to destroy a gaseous detector.

c) In a third and final step, all the remaining materials therefore underwent a series of aging tests. The final aging test was performed with all the materials, each one in a quantity 20 times larger (per straw) than foreseen for the real detector, placed in the outgassing box and heated up to $65^{\circ} \mathrm{C}$. The active gas passed through this box and then through a series of straws, which were irradiated with an X-ray beam $1 \mathrm{~cm}$ wide. These tests were performed with current densities of about $1 \mu \mathrm{A} / \mathrm{cm}$ and $0.1 \mu \mathrm{A} / \mathrm{cm}$ (the latter corresponding to the expected maximum current density in the straws at the LHC design 
luminosity). Typical relative gas gain variations as a function of accumulated charge are shown in Fig. 3.

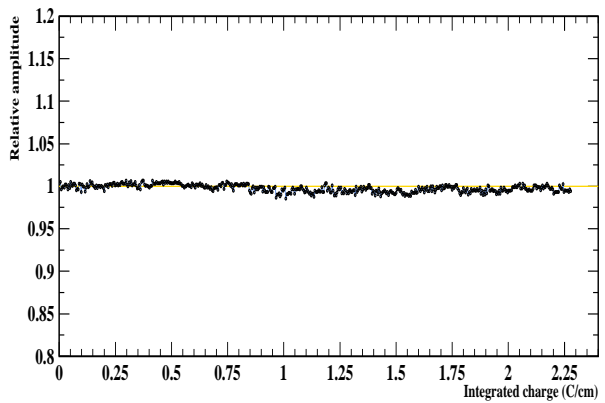

Fig. 3. TRT material tests: relative amplitude variation as a function of accumulated charge for a $1 \mu \mathrm{A} / \mathrm{cm}$ current density in the straw

No noticeable gas gain drop was observed under any of the test conditions. Some materials produced a detectable amount of hydrocarbon outgassing, but no wire deposits were observed after detailed wire analysis under an electron microscope. No indication of Si-deposits was found from G10 nor from FR4 materials, even if some of them smelled rather strongly after machining and irradiation (this outgassing was not detected by the gas chromatograph/mass spectrometer).

\section{Silicon deposits}

It is well known that the presence of volatile, Si-containing products (such as lubricants, mould-release agents or others) may lead to a very strong deposition process on the anode wires $[8,9]$. In $\mathrm{CF}_{4}$-containing gas mixtures, etching phenomena should occur in the avalanche region. Among the parameters, which govern the relative importance of these two processes, the most important ones are the amount of Si-containing products prone to creating deposits on the wire and the ionisation current density. For the chosen TRT gas mixture, Si-deposits have never been observed inside the irradiation area for ionisation current densities above $1 \mu \mathrm{A} / \mathrm{cm}$ because of the dominant etching activity induced by the $\mathrm{CF}_{4}$, but $\mathrm{Si}$ deposits are sometimes visible at the edges of the irradiation area. One should distinguish two sources of Si: internal (detector parts) and external (gas-system parts). The effects observed and the possible remedies to remove them are quite different for these sources.

\subsection{Internal sources of Si}

As described above, it has been shown during the selection and validation process of the TRT materials, that they do not produce any contamination, 
which may lead to the creation of deposits on the wire.

Nevertheless, first observations of the Si-deposits on the anode wire were made when operating at a low ionisation current density of $0.1 \mu \mathrm{A} / \mathrm{cm}$. A clean and validated gas system was used for the gas supply. It was very surprising when, for some straw samples, a very fast aging effect was observed already after 0.08 $\mathrm{C} / \mathrm{cm}$ of accumulated charge. After analysis of the internal straw surface, a large amount of Si was found, as shown in Figs. 4 and 5. It turned out to come from the straw manufacturing process (winding), for which a Si-containing lubricant was used by mistake in the early development stages. After a detailed review of the manufacturing process, a strict control was established at each step and the TRT straws were wound without any lubricant.

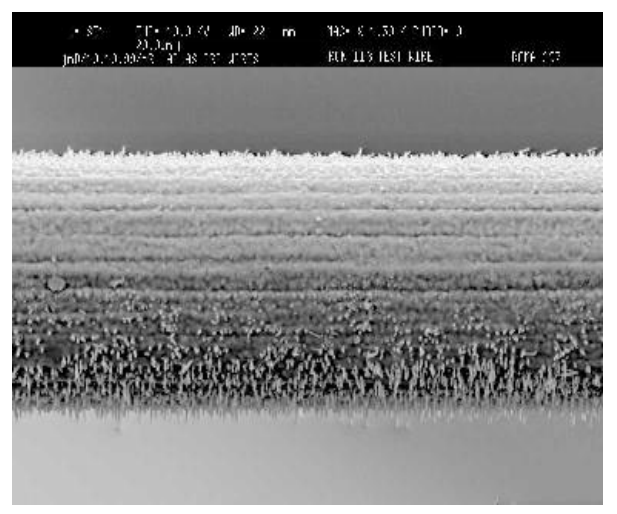

Fig. 4. Anode wire after $0.08 \mathrm{C} / \mathrm{cm}$ accumulated charge for a straw polluted by Si-lubricant. Such deposits lead to a gas gain drop of about $30 \%$.

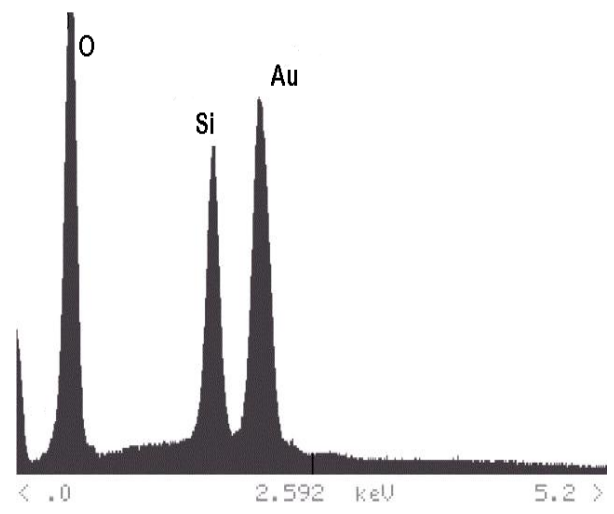

Fig. 5. EDX spectrum for deposit shown in Fig. 4. The peaks on the picture are from left to right: oxygen, silicon and gold.

However, the straw material itself (carbon coating) contains very small amounts of $\mathrm{Si}$, which may under specific conditions produce some aging effects. A typical EDX (Energy Dispersive X-ray) spectrum of the straw surface obtained during studies under the electron microscope is shown in Fig. 6. A very small Si-peak near the dominant-Al peak (Al-thickness of $0.1 \mu \mathrm{m}$ ) can be seen. The total thickness of $\mathrm{Si}$ is estimated to be a few molecular mono-layers. The height of the Si-peak varies very much from sample to sample, but never exceeds the value shown in Fig. 6. If such a straw is irradiated with a narrow beam of small intensity, one can observe a gas gain drop over a few hundred hours. An example of this behaviour is shown in Fig. 7: a test straw of $50 \mathrm{~cm}$ length was irradiated by a $1 \mathrm{~mm}$ wide X-ray beam $(6 \mathrm{keV})$ with an intensity of 5 $\mathrm{kHz}$ (corresponding to a current density of about $0.05 \mu \mathrm{A} / \mathrm{cm}$ ). The three curves correspond to different beam positions along the straw (separated by a distance of $150 \mathrm{~mm}$ ). The observed gas gain drop is the same for any beam position along the straw.

Silicon in the straw wall can only be present in a solid state (in the form of $\mathrm{SiO}$ or $\mathrm{SiO}_{2}$ ). It is impossible to provide an explicit identification of the chemical 
process through which this silicon can appear in the active gas. A reasonable hypothesis can be that active species, created in the avalanche plasma and containing fluorine atoms in different states, react with $\mathrm{Si}$ (or $\mathrm{SiO} / \mathrm{SiO}_{2}$ molecules) situated inside the cathode and produce volatile products, such as e.g. $\mathrm{SiF}_{4}$. These products are prone to deposit themselves on the anode wire when they arrive in the irradiation area. The straw area involved in this process can be rather large because of two effects:

-on one hand the active species travelling with the gas produce Si-containing volatile products downstream, and these products diffuse backwards to the ionisation area.

-on the other hand, the active species also diffuse upstream and produce Sicontaining molecules, which travel with the active gas and are delivered to the ionisation area.

For the chosen nominal gas flow in the TRT straws $\left(0.1 \mathrm{~cm}^{3} / \mathrm{min}\right)$, the diffusion effect can be as large as $5 \mathrm{~cm}$. This means that, even for a narrow ionisation beam, the straw area participating in the production of Si-containing products is rather large.

In order to evaluate better how this whole process occurs, we performed a few additional and more detailed tests. The same straws as used in the tests described above were irradiated by a beam of $2 \mathrm{~cm}$ width with a current density of $1 \mu \mathrm{A} / \mathrm{cm}$. First, the irradiation area was downstream and overlapped with a point of the straw where aging had been observed. After one day of irradiation, the signal amplitude in the aged point had recovered its original value (see

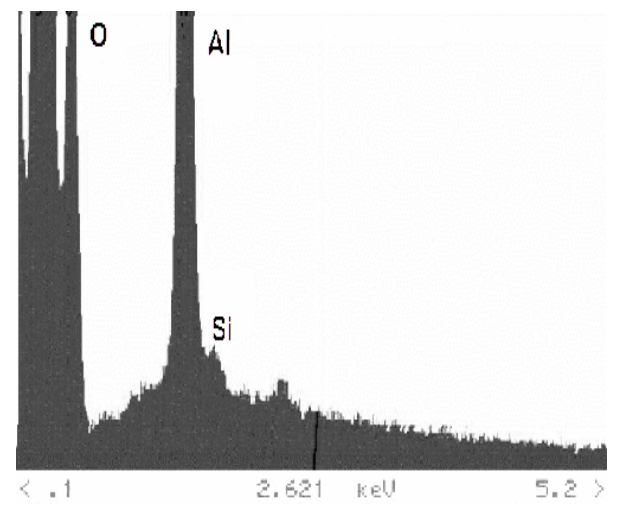

Fig. 6. EDX (Energy Dispersive X-ray) spectrum of the straw cathode surface for a straw produced without lubricant. The peak in the middle of the picture corresponds to $1000 \AA$ of $\mathrm{Al}$. A very small $\mathrm{Si}$ peak can barely be distinguished close to the right-hand side of the Al-peak.

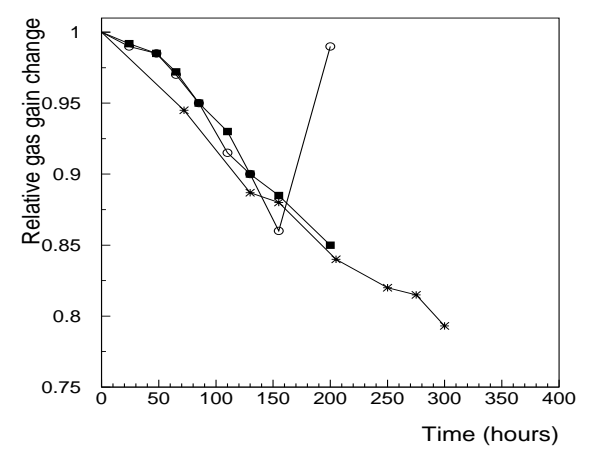

Fig. 7. Relative amplitude variation as a function of irradiation time with a $1 \mathrm{~mm}$ wide X-ray beam for three different beam positions along the straw. The last open circle shows the recovery obtained by irradiating that particular point in the straw with a $2 \mathrm{~cm}$ wide $\mathrm{X}$-ray beam and a current density of $1 \mu \mathrm{A} / \mathrm{cm}$ over less than 24 hours (see text). 
last open circle in Fig. 7). The narrow beam irradiation was then repeated at points situated $3 \mathrm{~cm}$ and $10 \mathrm{~cm}$ upstream of the cleaned area. No indication of any aging effect during at least 400 hours was observed for the nearest of the two points, whereas some aging was observed for the one farther away. Finally, the same treatment, with a $2 \mathrm{~cm}$ wide beam and a current density of $1 \mu \mathrm{A} / \mathrm{cm}$ during 20 hours, was carried out at the upstream part of the straw and the tests with a narrow beam were then repeated in a few places along the full $30 \mathrm{~cm}$ length of the straw. No aging effects were observed in any position along the straw over at least 600 hours of irradiation.

These tests prove first of all that the active species responsible for Si-cleaning survive for a rather long time and remove $\mathrm{Si}$ from the cathode surface as they travel with the active gas. A certain amount of diffusion of these active species is observed but is not sufficient to clean the upstream surface of the straw over distances more than a few $\mathrm{cm}$. In this particular case, the Si source in the straw is quite small in quantity and is removed after an accumulated charge of less than $3 \cdot 10^{-3} \mathrm{C}$ (about one day of LHC operation). The other important conclusion from these tests is that aging effects arising from the presence of such small amounts of Si in the straw may not be observed at all, if the irradiation area is wide, which will of course be the case during LHC operation. As a final confirmation that there should be no concern about operation at the LHC, the wide-beam test was carried out with a current density of $0.03 \mu \mathrm{A} / \mathrm{cm}$ (corresponding to initial operation at the LHC) on a straw, which had manifested aging effects in narrow beam tests. All the aged areas were under irradiation in this tests. After 70 hours of irradiation, not only had no aging been observed in the irradiation zone and its edges, but the deposits in the aged areas had been removed from the wire through the etching process.

In conclusion, although all straws contain traces of $\mathrm{Si}$, which have been observed under certain conditions to produce aging effect, such amounts of Si are not a problem under the real TRT operating conditions at the LHC, since they will be removed from the cathode wall after a few days of initial operation.

\subsection{External sources of Si}

One of the most complex issues for any gaseous detector is the cleanliness of the gas-supply system, especially in the case of a closed-circuit gas system, which is required for the TRT because of the high cost of the Xe gas. These systems normally contain many elements, and it is rather difficult to keep control of the manufacturing processes, which must guarantee the absence of polluting components. The most dangerous ones are Si-containing lubricants, which are unfortunately often used for the production of gas-system components. Quite 
different behaviours of straws exposed to such contaminants were observed depending on the amounts of contaminants:

a) In the case of a rather weak external source of $\mathrm{Si}$, all Si-containing molecules are stopped by the avalanche process and produce deposits only at the beginning of the irradiation area. Two examples of this type of behaviour are shown in Figs. 8 and 9. In both cases, some gas-system components under test were polluted by Si-containing lubricants or oil.

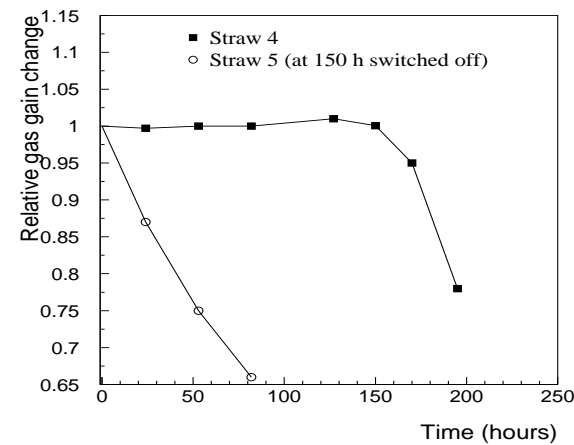

Fig. 8. Relative amplitude variation during irradiation with a narrow beam of 1 $\mathrm{mm}$ width where the gas flow is serially from straw 5 to straw 4 , and so on. The Si-source in this case is a lubricant used for the assembly of a flow regulator.

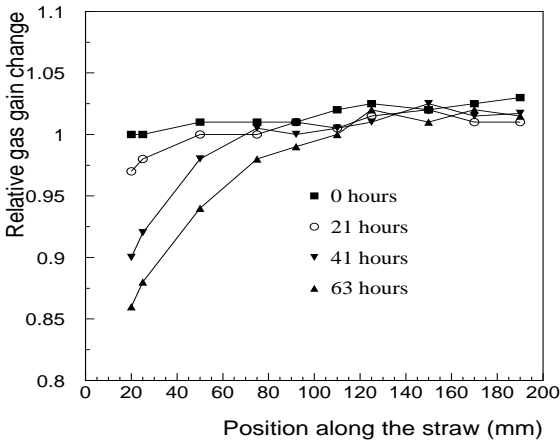

Fig. 9. Relative amplitude variation along the straw for different irradiation periods for a rather weak external source of Si. The straw was irradiated over its full length.

In the case of Fig. 8, five straws were connected serially and each of them was irradiated by a $1 \mathrm{~mm}$ wide $6 \mathrm{keV}$ X-ray beam $(5 \mathrm{kHz})$ near the middle of the straw. The active gas flow was from straw 5 to 4 , and so on. During the first 150 hours, all straws were connected to high voltage. Straw 5 showed significant aging immediately, but all the other straws remained stable. After 150 hours, straw 5 was disconnected from the high voltage (but left in the gas flow), and significant aging was observed immediately in straw 4 . The Si-source in this case was a lubricant used during flow-regulator assembly. The conclusion is that even a $1 \mathrm{~mm}$ wide irradiation area is sufficient to stop downstream Si-transport in this particular case. If the beam is wider, the amplitude degradation is observed first at the gas inlet, but it has a tendency to propagate further. This is shown in Fig. 9, where a $50 \mathrm{~cm}$ long straw was irradiated by a $40 \mathrm{~cm}$ wide beam, and, after 64 hours, a significant amplitude drop was observed up to a distance of $5 \mathrm{~cm}$ from the gas inlet. In this test, the main source of $\mathrm{Si}$ arose from pollution of the gas system by traces of oil.

b) In the case of a much stronger external source of Si (large pollution of the gas system by a Si-containing lubricant), there is not enough ionisation to stop all the $\mathrm{Si}$ and it is distributed along the straw. In this case, fast aging happens along the whole straw at the same time, as shown in Fig. 10 for a 
current density of $0.03 \mu \mathrm{A} / \mathrm{cm}$.

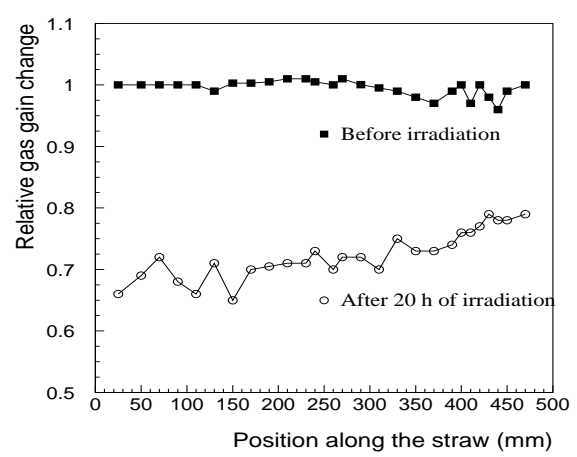

Fig. 10. Aging due to a strong external source of Si. The relative amplitude variation is shown as a function of the position along the straw before irradiation and after 20 hours of irradiation with an X-ray beam along the full length of the straw and at a current density of $0.03 \mu \mathrm{A} / \mathrm{cm}$.

It can be concluded from these tests that even weak external Si-sources are potentially very dangerous to the detector itself.

As already mentioned, the main source of $\mathrm{Si}$ in the gas systems arises from Sibased lubricant residuals. For instance, the case shown in Fig. 8 corresponds to a test, in which one of the standard flow regulators ("rotameter") was connected directly to the straw, as received from the manufacturer. Even a short exposure of the detector to such a component may very often leave residuals in the detector itself. For example, the use of this particular flow regulator for 70 hours resulted in a significant pollution of the straw: after connection to a clean gas supply line, the straw displayed an amplitude drop of $5 \%$ over 100 hours of irradiation with a narrow X-ray beam.

Unfortunately, the manufacturers do not or cannot always strictly control the production process for their equipment (except for the expensive components dedicated to the semiconductor industry). If the amount of lubricant is not very large, the equipment can be cleaned by ultrasonic cleaning: 15 minutes in Isopropanol (twice), then 15 minutes in distilled water. This method was used to clean the flow-regulator parts. After this procedure, no aging effect was observed during a few hundred hours of irradiation. Another effective method of cleaning was found to be flushing with DME gas, which is known to be a good solvent. Tests were conducted with the flow regulators and also with a small circulation gas-system, which had manifested aging before cleaning (in this case, Si may have come from mechanical flow regulators used in the system). After cleaning, no aging effects were observed in any of these systems during 400-600 hours of tests with a narrow X-ray beam. The minimal cleaning period needed for the flow regulators was found to be 20 hours with a DME gas flow of $0.8 \mathrm{l} / \mathrm{h}$. 
These measurements, as well as extensive past experience in this field, lead to the obvious conclusion that any gas system and its components should be carefully validated before they are commissioned with the real detector.

Some recommendations, which should help to guarantee the cleanliness of the gas-system can be listed as follows:

- Validate samples from the manufacturer in all cases;

- Specify and control the production process of all components as carefully as possible;

- Examine the parts carefully after delivery and carry out some aging tests with samples;

- If possible, clean parts ultrasonically;

- Verify the gas-system cleanliness in a dedicated test after assembly;

- Si-lubricant traces can be cleaned by DME-flushing after the gas system has been assembled (in this case the gas-system components should tolerate a short-term exposure to DME).

\section{Damage of the anode wire gold-plating}

Damage of the wire gold-plating was observed for the first time in 1996, during the straw wall irradiation studies with large doses (see section 2). In many cases, the gold surface was found to be destroyed, particularly at the edges of the irradiation region. Some tungsten-oxide (WO) deposits were observed outside the irradiation area. Similar effects were observed with gold-plated $\mathrm{Cu}$-Be wire. It was suspected that this effect may come from the large dose rate (a current density of $4 \mu \mathrm{A} / \mathrm{cm}$ was used in these tests).

A dedicated test was then performed under conditions close to those at the LHC. The prototype contained $611 \mathrm{~m}$-long straws connected in parallel to a gas manifold. 52 straws were included in a closed-loop circulating gas system and 9 straws were always flushed with fresh gas. The irradiation area was 60 $\mathrm{cm}$ wide and the dose rate was $0.7 \mu \mathrm{A} / \mathrm{cm}$ (only 6 times more than at the LHC). After $2 \mathrm{C} / \mathrm{cm}$ of accumulated charge, all straws showed a relative loss of amplitude of $2-3 \%$ and were opened for investigation. It was found that this effect is explained by a local increase of the wire diameter due to gold damage, as illustrated in Fig.11. This effect was observed for all the straws connected to both gas systems.

In order to understand this phenomenon, we performed dedicated studies under different conditions and for different types of wires (different manufacturers, different amount of gold, etc.). It was found that the way the gold-plating damage manifests itself is not very reproducible, and that it can even be dif- 


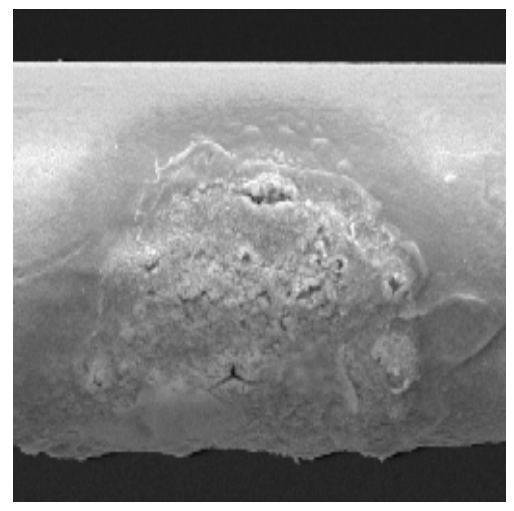

Fig. 11. Damage of the wire gold-plating after $2 \mathrm{C} / \mathrm{cm}$ of accumulated charge for a current density of $0.7 \mu \mathrm{A} / \mathrm{cm}$. Local deformation of the gold plating leads to an effective wire diameter increase.

ferent for the same type of wire during the same tests. The damage does not depend very much (except perhaps for the amount of observed WO deposit) on the exact gas composition (gas mixtures with $6 \%, 20 \%$ and $27.5 \%$ of $\mathrm{CF}_{4}$ were tested). The damage is not very sensitive to the gas gain nor to the amount of self-limited streamer (SLS) discharges (the gas gain was varied in these tests from $2 \cdot 10^{4}$ to $8 \cdot 10^{4}$; over this range the SLS rates increased by two orders of magnitude). The damage is also not very sensitive to the irradiation dose rate, which was varied from 0.7 to $5 \mu \mathrm{A} / \mathrm{cm}$, and appears at very different total accumulated charges, from 0.5 to $6 \mathrm{C} / \mathrm{cm}$, depending on the type of wire and on the operating conditions. Selected wire pictures from these tests are shown Fig. 12 as an illustration of the variety of gold-plating damage.

At higher dose rates, the damage is larger at the edges of the irradiation area. In some cases, active chemical processes under the gold-plating are clearly observed (the gold comes off in the form of chips). Wire swelling, or more precisely, swelling of the tungsten, has also been observed, but in contrast to the results reported in [10], this is not a very reproducible phenomenon: it happened just twice out of about 30 tests. In some tests, damage of the tungsten wire itself was observed, but these are rare occasions. The gold-plating layer very often becomes porous before further damage occurs. Tungsten oxide deposits are observed in certain cases, but not regularly; these deposits are most often observed outside the irradiation zone; they are sometimes very soft (they disappear under the electron beam during the electron microscope analysis), but sometimes they are hard. No fluorine-based deposits were observed at all.

In the tests described above, all the operating conditions were under control except for the amount of water and oxygen. Kapton is very transparent to $\mathrm{H}_{2} \mathrm{O}$, and water present in the ambient atmosphere therefore easily permeates through the straw wall. For a gas flow corresponding to one straw volume per hour, the water concentration inside the straw becomes almost immediately the same as the one outside ( $1-1.5 \%$ under normal conditions). 


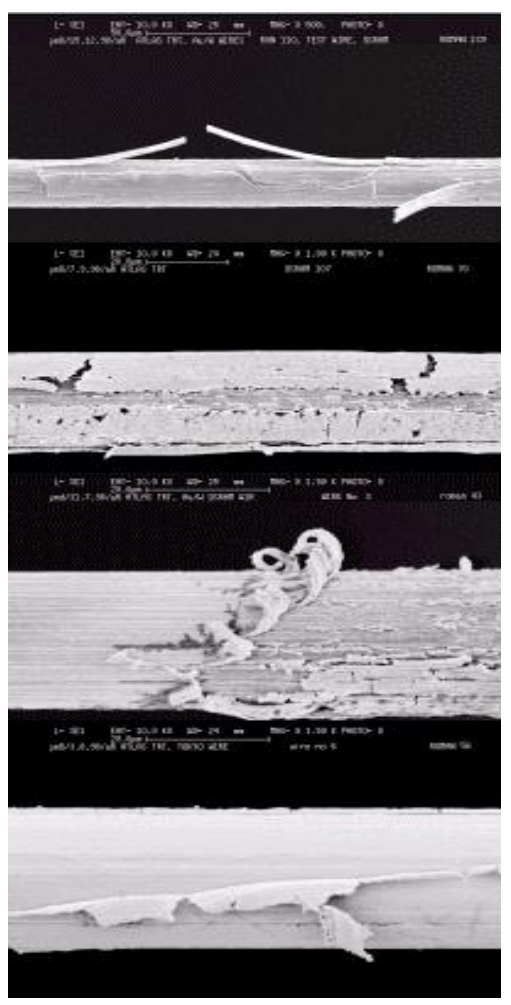

Fig. 12. Various manifestations of damage to gold plating on wire surface after 2-6 $\mathrm{C} / \mathrm{cm}$ of accumulated charge for different current densities $(1-4 \mu \mathrm{A} / \mathrm{cm})$.

In order to study the impact of the water and oxygen concentrations in the active gas on the gold-plating damage, we carried out a dedicated series of tests. The set-up was the same as the one described above, except that it contained three sets of straws and the gas system was open (no recirculation). All straws were placed in an $\mathrm{N}_{2}$ envelope and flushed with the standard gas mixture. For one set of straws, the input gas contained about $1.2 \%$ water, for the second set, it contained about $1.5 \%$ of Oxygen, and the third set acted as a reference set and was operated with the pure standard mixture.

The tests were carried out with a $4 \mu \mathrm{A} / \mathrm{cm}$ current density up to an accumulated dose of $6.5 \mathrm{C} / \mathrm{cm}$. The results of the wire analysis are shown in Figs. 13 and 14. The impact of water contamination is clearly observable in Fig. 13, whereas no gold-damage was observed for the other two sets of straws (pure gas mixture and gas mixture with $1.5 \%$ of Oxygen) as illustrated in Fig. 14.

Very interesting results were obtained when combining $\mathrm{H}_{2} \mathrm{O}$ and $\mathrm{O}_{2}$ in the gas mixture. The results were found to depend very strongly on the type of wire. Figs. 15, 16 and 17 display the results obtained for the standard gas mixture with an additional $1.2 \%$ of $\mathrm{H}_{2} \mathrm{O}$ and $1.5 \%$ of $\mathrm{O}_{2}$ and for another wire manufacturer (OSRAM). A huge amount of WO deposits were found on top of the gold-plating already after $0.5 \mathrm{C} / \mathrm{cm}$ of accumulated charge. These WO deposits exist as an amorphous substance (Fig. 15) or even as a crystalline 
structure (Fig. 17). In spite of this large observed amount of WO deposits, no significant gold-plating damage was observed for this accumulated charge. One can clearly distinguish the undamaged gold surface in the cracks of WO deposits in Fig. 17.

If the water content is kept at a low level, the damage to the gold-plating is minimal. Fig. 18 shows a wire surface (Toshiba), tested with the standard mixture and an additional $0.4 \%$ of water after $5 \mathrm{C} / \mathrm{cm}$ of accumulated charge (the current density in this test was $1 \mu \mathrm{A} / \mathrm{cm}$ ). For water concentrations below $0.1 \%$, no gold-plating damage is observed up to $20 \mathrm{C} / \mathrm{cm}$ of accumulated charge, as illustrated in Fig. 19. Last tests were carried out with a current

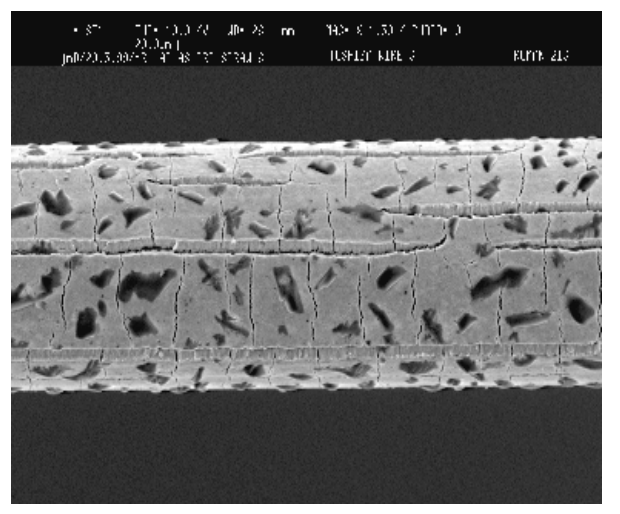

Fig. 13. Damage to gold-plating on wire surface observed after $6.5 \mathrm{C} / \mathrm{cm}$ of accumulated charge for the wire manufacturer chosen for the TRT (Toshiba). The gas mixture was the standard one with an additional $1.2 \%$ of water.

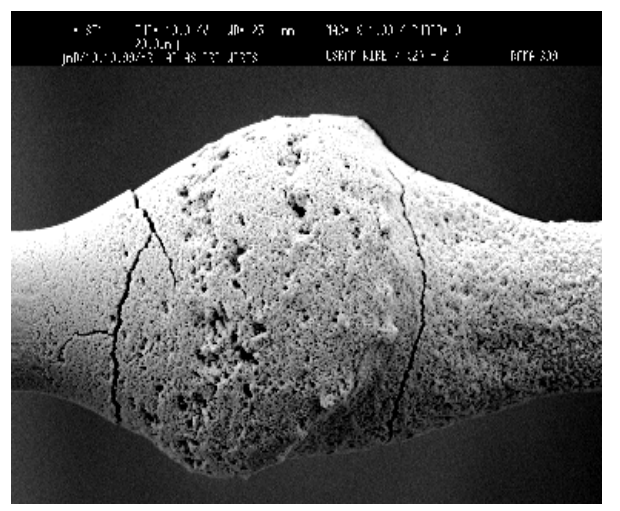

Fig. 15. Surface of wire (OSRAM) observed after $0.5 \mathrm{C} / \mathrm{cm}$ of accumulated charge, for the standard gas mixture with $1.2 \%$ of water and $1.5 \%$ of oxygen added. Amorphous deposits of WO are observed (see Fig 16).
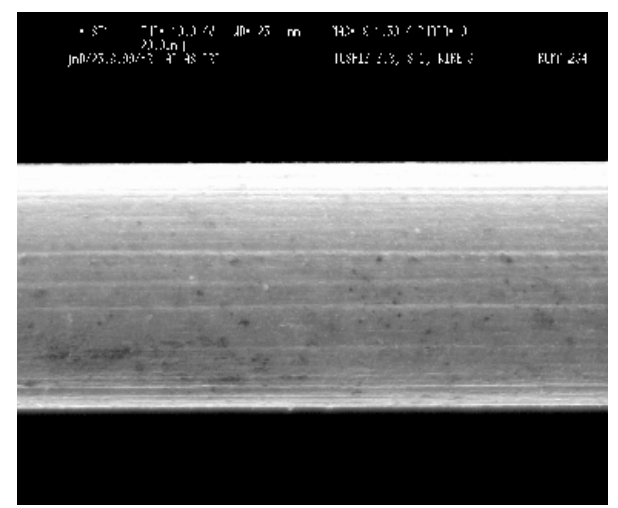

Fig. 14. The same as in Fig. 13 for the standard gas mixture with an additional $1.5 \%$ of Oxygen. No gold damage is observed.

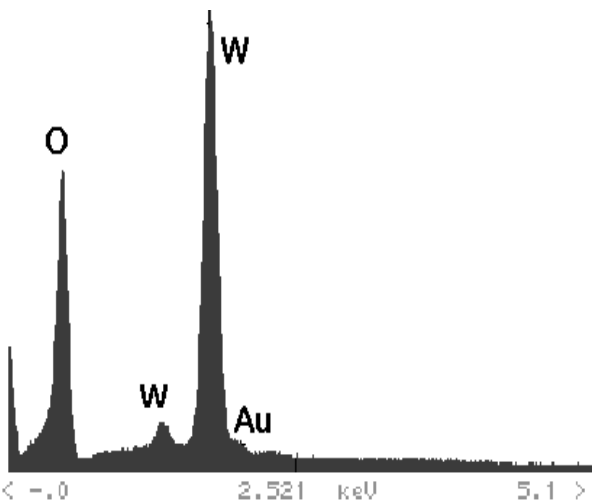

Fig. 16. EDX (Energy Dispersive X-ray) spectrum of the deposit on the wire shown in Fig 15. The two largest peaks correspond to oxygen (left) and tungsten (right). 


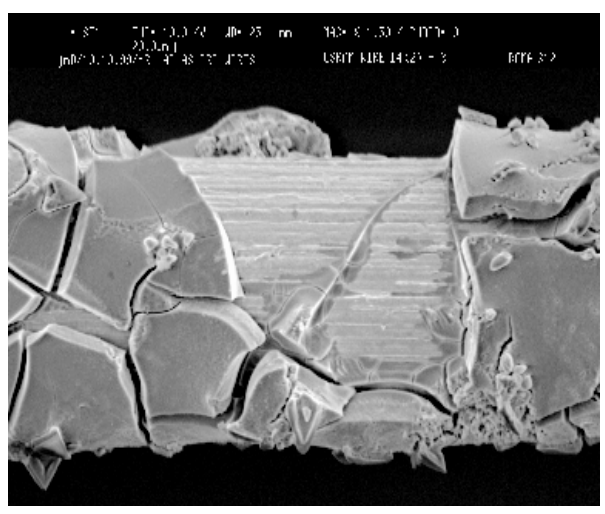

Fig. 17. The same as in Fig 15 for a different wire sample: crystalline deposits of WO are observed.

density of 4-5 $\mu \mathrm{A} / \mathrm{cm}$, and some of them were repeated with $1 \mu \mathrm{A} / \mathrm{cm}$ up to $10 \mathrm{C} / \mathrm{cm}$ of accumulated charge. The result was the same: no gold-plating damage is observed for any type of wire if the water content is below $0.1 \%$.

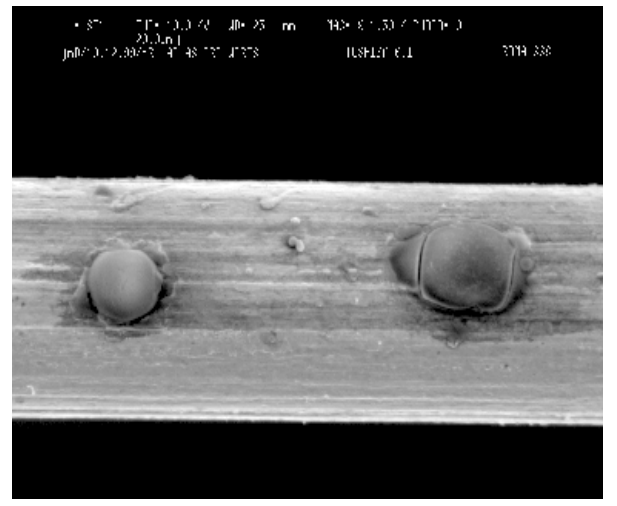

Fig. 18. Toshiba wire surface after 5 $\mathrm{C} / \mathrm{cm}$. Gas composition: standard mixture $+0.4 \%$ of $\mathrm{H}_{2} \mathrm{O}$.
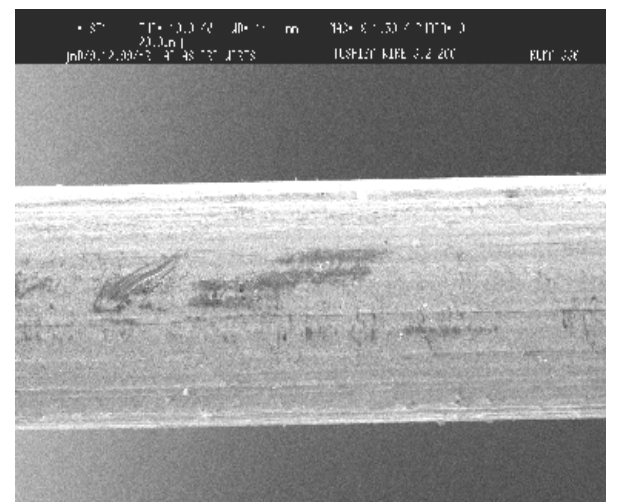

Fig. 19. Toshiba wire surface after 20 $\mathrm{C} / \mathrm{cm}$. Gas composition: standard mixture, water concentration less then $0.1 \%$

In conclusion, the main agent responsible for the gold-plating damage on the wire surface are active radicals (products of $\mathrm{CF}_{4}$ disintegration, particularly F) and $\mathrm{H}_{2} \mathrm{O}$, which together create $\mathrm{HF}$ acid. The presence of oxygen speeds up wire degradation process (mainly due to fast creation of solid WO deposits). These active radicals do not produce any damage in the absence of water. The damage itself very strongly depends on the water concentration and is rather small for water concentrations below 0.4\%. Similar problems should be expected with a $\mathrm{C}_{2} \mathrm{H}_{2} \mathrm{~F}_{4}$ gas, even without any $\mathrm{H}_{2} \mathrm{O}$ contamination. There is no indication that $\mathrm{F}$ creates any solid deposits with any straw material, although it is known that it reacts with $\mathrm{Au}, \mathrm{Xe}, \mathrm{W}$ and $\mathrm{O}$. In these particular tests, all products of chemical reactions with $\mathrm{F}$ are volatile (like $\mathrm{WF}_{6}$ ) or they react with other components, thereby creating volatile products. Possibly, HF plays the role of a catalyst in the chemical processes near the wire. No damage has been observed at water concentrations below $0.1 \%$ for all combinations of gases $\left(\mathrm{Xe}, \mathrm{CO}_{2}, \mathrm{CF}_{4}, \mathrm{O}_{2}\right)$ and for all wire types at current densities above 1 
$\mu \mathrm{A} / \mathrm{cm}$.

The type of damage and the rapidity with which it appears depend on the wire manufacturing process and the choice of the gold plating thickness (the damage appears faster for gold-plating produced with less adhesion to the $\mathrm{W}$ alloy, the addition of Ni-plating increases the rate of damage, etc.)

After completion of all the gold-plating robustness tests, Toshiba was chosen as the manufacturer and the gold plating was specified as 7-9\% (in weight).

\section{Active radicals}

As shown in the introduction, the total ionisation current in the TRT at the LHC design luminosity is about $3 \mathrm{~A}$. Active species produced in the avalanche may therefore be present in sufficient quantities to seriously attack and damage the materials, which are inside the detector or in the gas system itself. The studies described in the previous sections have demonstrated that, under controlled conditions, the effect of these active species on the straw itself is small and that the straw and wire survive at least up to accumulated charges of $20 \mathrm{C} / \mathrm{cm}$ (20 years of LHC operation).

In order to evaluate how serious the impact of these active species might be on the other materials situated outside the avalanche region we carried out a special test under very harsh conditions. The test set-up consisted of a special discharge device installed in a closed-loop gas system, which produced active radicals through glow discharges, a box with tested materials situated downstream of the discharge device, a purification system and a circulation pump. The purification system contained three components: charcoal, molecular sieves and a Cr-Ni catalyst. After about 300 hours of circulation with a glow discharge current of 4-5 mA, the box was opened and the material surfaces were examined. The most striking results for parts of printed-circuit board, which were in the box, are shown in Figs. 20 and 21. Some $\mathrm{SiO} / \mathrm{SiO}_{2}$ crystals were found on the gold-plated surfaces of a printed-circuit board (Fig. 20) and the coverlay from this printed-circuit board was partially etched off (Fig. 21). It is not totally clear where the $\mathrm{Si}$ comes from. Possibly, active species reacting with some of the Si-containing materials (for instance, with the glass of the printed-circuit boards or with the molecular sieves) produce volatile products, which travel back to the avalanche plasma region and create dipole molecules able to make crystalline deposits even outside the irradiation area. It is very difficult to extrapolate from such tests to the LHC situation because the glow discharge conditions are very different from those in the proportional amplification region (different electric fields, different electron energies, different plasma densities, etc.). In any event, these tests were performed under much 
harsher conditions than at the LHC, and their results should be considered as some sort of warning, implying that the problem of active species should be understood and resolved before LHC operation starts.

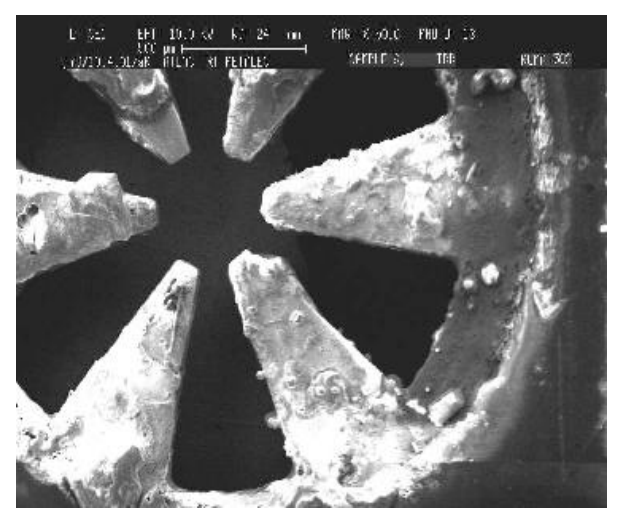

Fig. 20. $\mathrm{SiO} / \mathrm{SiO}_{2}$ deposits on the gold-plated surface of a printed-circuit board after 300 hours of glow discharge tests (see text).

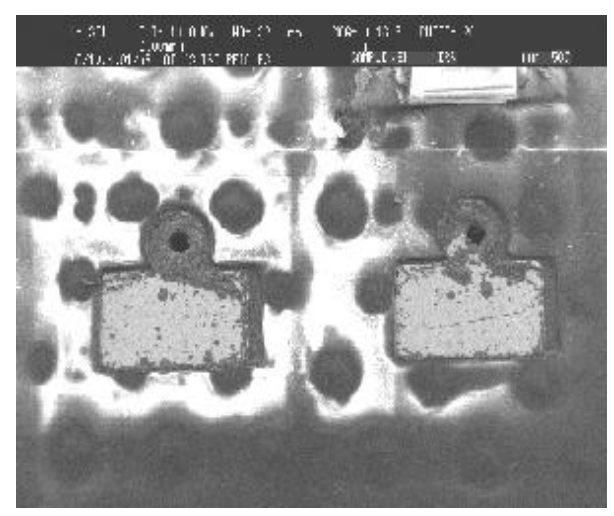

Fig. 21. Printed-circuit board with coverlay partially etched off after 300 hours of glow discharge tests (see text).

\section{Conclusions}

After 12 years of R\&D on the ATLAS TRT straws, on the detector design and material choices and the gas system itself, most of the problems encountered have been solved: the straw cathode and wire survive accumulated charges of $20 \mathrm{C} / \mathrm{cm}$ (20 years of LHC operation), the detector materials have all been carefully validated in terms of outgassing and Si-pollution, and some of the gassystem components have been chosen and validated. Nevertheless, much work still remains to be done concerning the final choices of the purification system and the understanding of the impact of long-lived active radicals present in the chosen gas mixture, when operating in a closed-circuit gas system.

The experience and results collected to-date can be summarised as follows:

- The straw cathode material is only slightly affected by active species up to $18 \mathrm{C} / \mathrm{cm}$ accumulated, and the reaction products do not produce any deposits on the wire.

- The materials chosen for the TRT construction do not produce any contamination leading to deposits on the wire, provided all Si-containing materials are not in direct contact with active radicals.

- There are two competitive processes near the wire: polymerisation and etching. The resulting balance has been studied in detail in the case of Sicontamination and found to be very sensitive to the Si-source intensity and to the ionisation current density. 
- There are traces of Si inside the straw material itself (a few atomic monolayers), but under real LHC operating conditions, this Si will not produce deposits on the wire.

- External sources of Si are potentially very dangerous. Even a rather weak but constant source of Si produces deposits near the gas inlet, which then have a tendency to propagate along the wire.

- One of the major sources of external Si arises from lubricant residuals in the gas-system components. These residuals can be removed by ultrasonic cleaning procedures and sometimes by flushing DME gas through the system.

- Statements by manufacturers that their products do not contain lubricants have been found to be inaccurate and a validation procedure must be foreseen for each element.

- The observed wire gold damage phenomena have quite complicated signatures, but do not occur at ionising current densities above $1 \mu \mathrm{A} / \mathrm{cm}$ if the amount of water in the active gas does not exceed 1000 ppm. Water most probably reacts with the F-containing radicals produced in the avalanche, creating HF acid, which is responsible for the destruction of the gold-plating.

- Active species created in the avalanche live for a rather long time and may attack the detector and gas-system components. Therefore, special purification elements for the removal of these active species from the closed-loop gas system may have to be foreseen.

\section{Acknowledgements}

The research described in this publication was partly made possible thanks to the following funding agencies: the European Union (DGXII), the International Science Foundation (grant NM5J000), the Swedish Natural Science Research Council, the Swedish Council for Planning and Coordination of Research, the State Committee for Scientific Research, Poland (grant 620/E77/SPUB-M/CERN/P-03/DZ295/2000-2002), the International Science and Technology Center (ISTC poject 441-99).

\section{References}

[1] ATLAS Inner Detector Technical Design Report, CERN/LHCC/97-17, ATLAS TDR 5, 30 April 1997.

[2] V. Bondarenko, et al., Nucl. Instr. and Meth. A 327 (1993) 386.

[3] T. Akesson, et al., Nucl. Instr. and Meth. A 361 (1995) 440. 
[4] V. Bondarenko, et al., CERN Preprint, CERN-PPE/91-191(1991).

[5] M. Capeans, et al., Nucl. Instr. and Meth. A 337 (1993) 122.

[6] A. Romaniouk, ATLAS Internal Note, ATL-INDET-98-211(1998).

[7] F. Guarino, et.al., ATLAS Internal Note, ATL-INDET-99-011(1999).

[8] J. Va'vra, Nucl. Instr. and Meth. A 252 (1986) 547.

[9] J. A. Kadyk, Nucl. Instr. and Meth. A 300 (1991) 436.

[10] T. Ferguson, et al., Swelling phenomena in an anode wire aging under a high accumulated dose, these proceedings. 